\title{
CHAINS OF STRONGLY NON-REFLEXIVE DUAL GROUPS OF INTEGER-VALUED CONTINUOUS FUNCTIONS
}

\author{
HARUTO OHTA
}

(Communicated by Franklin D. Tall)

Dedicated to Professor Shōzō Sasada on his 60th birthday

\begin{abstract}
Answering a question of Eklof-Mekler (Almost free modules, settheoretic methods, North-Holland, Amsterdam, 1990), we prove: (1) If there exists a non-reflecting stationary set of $\omega_{i}$ consisting of ordinals of cofinality $\omega$ for each $1<i<\omega$, then there exist abelian groups $A_{n}(n \in \mathbb{Z})$ such that $A_{n}^{*} \cong A_{n+1}$ and $A_{n} ¥ A_{n+2}$ for each $n \in \mathbb{Z}$. (2) There exist abelian groups $A_{n}(n \in \mathbb{Z})$ such that $A_{n}^{*} \cong A_{n+1}$ for each $n \in \mathbb{Z}$ and $A_{n} ¥ A_{n+2}$ for each $n<0$. The groups $A_{n}$ are the groups of $\mathbb{Z}$-valued continuous functions on a topological space and their dual groups.
\end{abstract}

\section{INTRODUCTION}

For an abelian group $A$, let $A^{*}$ denote the $\mathbb{Z}$-dual group of $A$, i.e., $A^{*}=$ $\operatorname{Hom}(A, \mathbb{Z})$, where $\mathbb{Z}$ is the group of integers. An abelian group $A$ is said to be reflexive if the canonical homomorphism $A \rightarrow A^{* *}$ is an isomorphism, and $A$ is said to be strongly non-reflexive if $A$ is not isomorphic to $A^{* *}$. In [4, p.455], Eklof and Mekler asked: Is there a $\mathbb{Z}$-chain of strongly non-reflexive dual groups, i.e., abelian groups $A_{n}(n \in \mathbb{Z})$ such that for all $n, A_{n}^{*}=A_{n+1}$ and $A_{n}$ is not isomorphic to $A_{n+2}$ ? The purpose of this note is to give a consistency answer and a partial answer to this question by using the abelian groups $C(X, \mathbb{Z})$ of continuous functions from a topological space $X$ to the discrete group $\mathbb{Z}$. For a regular, uncountable cardinal $\kappa$, let $E(\kappa)$ be the following statement: There exists a non-reflecting stationary set of $\kappa$ consisting of ordinals of cofinality $\omega$. Here, a subset $E$ of $\kappa$ is non-reflecting if $E \cap \lambda$ is not stationary in $\lambda$ for each ordinal $\lambda<\kappa$ with cofinality $>\omega$. Obviously, $E\left(\omega_{1}\right)$ is true. It is known (cf. [4]) that under $V=L, E(\kappa)$ holds for any regular, not weakly compact cardinal $\kappa$. In section 3 , we prove:

Theorem 1. If $E\left(\omega_{i}\right)$ holds for each $1<i<\omega$, then there exist topological spaces $X_{n}(n \in \mathbb{Z})$ such that, if $A_{2 n}=C\left(X_{n}, \mathbb{Z}\right)$ and $A_{2 n+1}=A_{2 n}^{*}$ for each $n \in \mathbb{Z}$, then $A_{n}^{*} \cong A_{n+1}$ and $A_{n} ¥ A_{n+2}$ for each $n \in \mathbb{Z}$.

Received by the editors July 6, 1994.

1991 Mathematics Subject Classification. Primary 54C40, 20K20, 20 K45.

Key words and phrases. Abelian group, continuous function, dual group, reflexivity, strong non-reflexivity, $\mathbb{Z}$-compact.

Research partially supported by Grant-in-Aid for Scientific Research No. 06640125, Ministry of Education, Science and Culture. 
Theorem 2. There exist topological spaces $X_{n}(n \in \mathbb{Z})$ such that, if $A_{2 n}=$ $C\left(X_{n}, \mathbb{Z}\right)$ and $A_{2 n+1}=A_{2 n}^{*}$ for each $n \in \mathbb{Z}$, then $A_{n}^{*} \cong A_{n+1}$ for each $n \in \mathbb{Z}$ and $A_{n} ¥ A_{n+2}$ for each $n<0$.

Strongly non-reflexive dual groups were constructed by several authors ([3], [4], [5] and [6]) and, in particular, Sageev-Shelah and Eklof-Mekler-Shelah [5] constructed an $\omega$-chain of strongly non-reflexive dual groups assuming $\diamond_{\omega_{1}}$. The history of non-reflexive dual groups can be found in [4] and [5].

Throughout the paper, by a group we mean an abelian group and by a space we mean a 0 -dimensional $T_{0}$-space, i.e., a topological space with a base $\mathcal{B}$, consisting of clopen (= closed and open) sets, such that $\bigcap\{U: x \in U \in \mathcal{B}\}=\{x\}$ for each $x \in X$. Suppose that $\left\{X_{\alpha}\right\}_{\alpha \in A}$ is a collection of spaces. Take a copy $X_{\alpha}^{\prime}$ of $X_{\alpha}$ for each $\alpha$ so that $X_{\alpha}^{\prime} \cap X_{\beta}^{\prime}=\emptyset$ if $\alpha \neq \beta$. Let $X=\bigcup_{\alpha \in A} X_{\alpha}^{\prime}$ and define a topology for $X$ by declaring a subset $U \subset X$ to be open if and only if $U \cap X_{\alpha}^{\prime}$ is open in $X_{\alpha}^{\prime}$ for each $\alpha \in A$. The space $X$ with this topology is called the discrete sum of $\left\{X_{\alpha}\right\}_{\alpha \in A}$, and is denoted by $\bigsqcup_{\alpha \in A} X_{\alpha}$, or by $X_{1} \sqcup X_{2}$ if $A=\{1,2\}$. The cardinality of a set $A$ is denoted by $|A|$. For ordinals $\lambda$ and $\mu$ with $\lambda<\mu$, we write $(\lambda, \mu]=\{\alpha: \lambda<\alpha \leq \mu\}$ and $[\lambda, \mu)=\{\alpha: \lambda \leq \alpha<\mu\}$; as usual, we also write $\mu$ for $[0, \mu)$. Other terminology and notation will be used as in [4].

\section{Preliminaries}

We state two lemmas, which play key roles in our proof of Theorems 1 and 2, together with some definitions. Except for Lemma 2 below, each definition and result stated here appears in [2] or [4]. A space $X$ is called $\mathbb{Z}$-compact if it is homeomorphic to a closed subspace of a product $\mathbb{Z}^{\kappa}$ for some cardinal $\kappa$. It is known that each space $X$ can be densely embedded in a unique $\mathbb{Z}$-compact space $\beta_{\mathbb{Z}} X$ to which each $\mathbb{Z}$-valued continuous function on $X$ admits a continuous extension. By the property of $\beta_{\mathbb{Z}} X$, the groups $C(X, \mathbb{Z})$ and $C\left(\beta_{\mathbb{Z}} X, \mathbb{Z}\right)$ are isomorphic. A $\mathbb{Z}$ valued function on a space $X$ is said to be $k$-continuous if the restriction $f \mid K$ is continuous for each compact set $K$ of $X$. Let $k_{\mathbb{Z}} X$ be the set $X$ equipped with the smallest topology so that every $\mathbb{Z}$-valued $k$-continuous function on $X$ is continuous.

Lemma 1 ([1, Lemma 4.4]). If $X$ is a $\mathbb{Z}$-compact space and no compact set of $X$ is of measurable cardinality, then $C(X, \mathbb{Z})^{* *}$ is isomorphic to $C\left(k_{\mathbb{Z}} X, \mathbb{Z}\right)$.

For a space $X$, let $w(X)=\omega \cdot \min \{|\mathcal{B}|: \mathcal{B}$ is a base of $X\}$.

Lemma 2 ([3, Theorem 3.1]). Let $X$ be a space and $\kappa$ an infinite cardinal. Then there exists a subgroup of $C(X, \mathbb{Z})^{*}$ isomorphic to $\mathbb{Z}^{\kappa}$ if and only if there exists a compact set $K$ of $\beta_{\mathbb{Z}} X$ with $w(K) \geq \kappa$.

A clopen ultrafilter on a space $X$ is a collection $p$ of clopen sets in $X$ which has the finite intersection property and has the property that for every clopen set $U$ in $X$, exactly one of $U$ and $X \backslash U$ is in $p$. A clopen ultrafilter $p$ is $\omega_{1}$-complete if $p$ intersects with each countable cover of $X$ consisting of clopen sets, and $p$ converges to a point $x$ if $\bigcap\{V: V \in p\}=\{x\}$. A clopen ultrafilter which converges is also called principal. It is known (cf. [2] or [4]) that a space $X$ is $\mathbb{Z}$-compact if and only if every $\omega_{1}$-complete clopen ultrafilter on $X$ converges. It follows that the discrete sum $\bigsqcup_{\alpha \in A} X_{\alpha}$ of $\mathbb{Z}$-compact spaces $X_{\alpha}$ is $\mathbb{Z}$-compact when $|A|$ is non-measurable.

The notion of $\mathbb{Z}$-compact spaces originated with Mrówka (see the references of [2]); many topologists use the term ' $N$-compact' instead of ' $\mathbb{Z}$-compact'. 


\section{Proof of Theorems 1 And 2}

Throughout this section, let $\kappa$ be a regular, uncountable, non-measurable, successor cardinal and $\Omega$ the set of all ordinals less than $\kappa$ which are successors or limits of cofinality $\omega$. For a subset $X$ of $\kappa+1$, we call the subspace topology on $X$ induced from the order topology the relative order topology. For a set $X$ with $\kappa \in X \subset \kappa+1$ and a topology $\tau$ on $X$, we call the space $(X, \tau)$ an adequate space if it satisfies the following conditions (1)-(4):

(1) $\tau$ is finer than the relative order topology on $X$.

(2) $\{(\lambda, \kappa] \cap X: \lambda<\kappa\}$ is a neighborhood base of the point $\kappa$ in $(X, \tau)$.

(3) Each point of $X \cap \Omega$ has a countable neighborhood base in $(X, \tau)$.

(4) $\beta_{\mathbb{Z}}((X \cap \Omega) \cup\{\kappa\})=(X, \tau)$, where $(X \cap \Omega) \cup\{\kappa\}$ is a subspace of $(X, \tau)$.

Note that (4) implies that an adequate space is $\mathbb{Z}$-compact. For a space $Y$, define $k w(Y)=\sup \{w(K):$ compact $K \subset Y\}$. For spaces $X$ and $Y$, we write $X \approx Y$ if $X$ is homeomorphic to $Y$.

Lemma 3. Assume that $E(\kappa)$ holds and let $E$ be a non-reflecting, stationary and co-stationary set of $\kappa$ with $E \subset \Omega$. Let $X=(X, \tau)$ be an adequate space. Then there exists a topology $v$ on $E \cup\{\kappa\}$ such that the following holds for the space $Y=(E \cup\{\kappa\}, v)$ :

(a) $Y$ is an adequate space.

(b) $k w(Y)=\omega$.

(c) $k w\left(\beta_{\mathbb{Z}} k_{\mathbb{Z}} Y\right)=k w(X)$.

(d) $\beta_{\mathbb{Z}} k_{\mathbb{Z}} \beta_{\mathbb{Z}} k_{\mathbb{Z}} Y \approx \beta_{\mathbb{Z}} k_{\mathbb{Z}} Y \sqcup \beta_{\mathbb{Z}} k_{\mathbb{Z}} X$.

Proof. Since $E$ can be decomposed into $\kappa$ many disjoint stationary sets, we can write $E=\bigcup\left\{E_{\lambda}: \lambda \in(X \cap \Omega) \cup\{\kappa\}\right\}$, where each $E_{\lambda}$ is stationary and $E_{\lambda} \cap E_{\mu}=\emptyset$ for $\lambda \neq \mu$. Moreover, we can assume that

$$
\lambda \notin E_{\lambda} \text { for each } \lambda \in X \cap \Omega .
$$

Define a map $\varphi: E \rightarrow \Omega$ by $\varphi(\alpha)=\lambda$ if $\alpha \in E_{\lambda}$ for $\lambda \in X \cap \Omega$ and $\varphi(\alpha)=\alpha$ if $\alpha \in E_{\kappa}$. Put $G=\{\langle\alpha, \varphi(\alpha)\rangle: \alpha \in E\}, H=\{\langle\kappa, \lambda\rangle: \lambda \in X\}$ and $S=G \cup H(\subset$ $(E \cup\{\kappa\}) \times(\kappa+1))$. Topologize $S$ as follows: A basic neighborhood of $\langle\alpha, \lambda\rangle \in S$ for $\alpha \notin E_{\kappa}$ is $((\beta, \alpha] \times U) \cap S$ for $\beta<\alpha$ and an open neighborhood $U$ of $\lambda$ in $X$ such that $(\beta, \alpha] \cap U=\emptyset$, and a basic neighborhood of $\langle\alpha, \alpha\rangle \in S$ for $\alpha \in E_{\kappa}$ is $((\beta, \alpha] \times(\beta, \alpha]) \cap S$ for $\beta<\alpha$. By (1), (2) and (5), this defines a topology on $S$. Let $T$ be the quotient space obtained from $S$ by collapsing the set $H$ to the point $\langle\kappa, \kappa\rangle$ and $\psi: S \rightarrow T$ the quotient map. Then there is a bijection $h: T \rightarrow E \cup\{\kappa\}$ such that $h \circ \psi=\pi$, where $\pi: S \rightarrow E \cup\{\kappa\}$ is the projection. Define $v$ to be the topology on $E \cup\{\kappa\}$ which makes the map $h$ a homeomorphism and put $Y=(E \cup\{\kappa\}, v)$.

To prove (a)-(d), let $\gamma$ denote the relative order topology on $E \cup\{\kappa\}$. We begin by proving (a). By the definition, $\gamma \subset v$, and hence $Y$ satisfies (1). By using (2) for $X$ and the regularity of $\kappa$, it is easily checked that every open set in $S$ including $H$ includes an open set of the form $((\mu, \kappa] \times(\kappa+1)) \cap S$ for some $\mu<\kappa$. This implies that $\mathrm{Y}$ satisfies (2). By the fact that $E \subset \Omega$ and (3) for $X$, each point of $G$ has a countable neighborhood base. Hence, $Y$ satisfies (3). Since $E \subset \Omega$, $Y=(Y \cap \Omega) \cup\{\kappa\}$. Thus, if we show that $Y$ is $\mathbb{Z}$-compact, then (4) would be satisfied. To do this and to prove Claim 4 below, let $Y_{\mu}=Y \cap \mu$ for each $\mu<\kappa$.

Claim 1. For each $\mu<\kappa, Y_{\mu}$ is $\mathbb{Z}$-compact. 
Proof. In case $\kappa=\omega_{1}$, this is obvious since each $Y_{\mu}$ is then countable. So we prove the case $\kappa>\omega_{1}$ by induction. Assume that $\mu<\kappa$ and $Y_{\lambda}$ is $\mathbb{Z}$-compact for each $\lambda<\mu$. Since a union of a $\mathbb{Z}$-compact space and a singleton is $\mathbb{Z}$-compact, $Y_{\mu}$ is $\mathbb{Z}$-compact if $\mu$ is a successor. If $\mu$ is a limit, then either cofinality $\mu=\omega$ or $Y_{\mu}$ is not stationary in $\mu$, because $E$ is non-reflecting. In each case, since $\gamma \subset v$, we can write $Y_{\mu}=\bigsqcup_{\xi \in \Xi} A_{\xi}$, where each $A_{\xi}$ is bounded in $\mu$ and clopen in $Y$. By our assumption, each $A_{\xi}$ is $\mathbb{Z}$-compact. Since $\kappa$ is non-measurable, $Y_{\mu}$ is $\mathbb{Z}$-compact. $\square$

To prove that $Y$ is $\mathbb{Z}$-compact, let $p$ be an $\omega_{1}$-complete clopen ultrafilter on $Y$. If $p$ does not converge to $\kappa$, then $\kappa \notin U$ for some $U \in p$. Since $Y$ satisfies (2), there is $\mu<\kappa$ such that $U \subset Y_{\mu}$. By Claim 1,p converges to a point of $U$. Thus $Y$ is $\mathbb{Z}$-compact and hence satisfies (4).

Claim 2. Every compact set of $Y$ is countable.

Proof. Suppose that $K \subset Y$ is compact and $|K|>\omega$. Then there is a strictly increasing sequence $\left\{\lambda_{\alpha}: \alpha<\omega_{1}\right\}$ in $K$. Put $\lambda=\sup \lambda_{\alpha}$. Since $\gamma \subset v, K$ is compact in $\gamma$, and hence, $K \cap \lambda$ is closed and unbounded in $\lambda$ with respect to its relative order topology. If $\kappa=\omega_{1}$, then $\lambda=\omega_{1}$ and this contradicts the fact that $E$ is co-stationary. If $\kappa>\omega_{1}$, this contradicts the fact that $E$ is non-reflecting.

The statement (b) follows from Claim 2 and (2), (3) for $Y$.

Claim 3. $k_{\mathbb{Z}} Y \approx G$ and $k_{\mathbb{Z}} S \approx G \sqcup k_{\mathbb{Z}} X$.

Proof. By Claim 2 and (2) for $Y$, the characteristic function of $E$ on $Y$ is $k$ continuous, which implies that $k_{\mathbb{Z}} Y \approx k_{\mathbb{Z}} E \sqcup\{\kappa\}$. Since each point of $E$ has a countable neighborhood base, $k_{\mathbb{Z}} E=E$. Since $E \sqcup\{\kappa\} \approx E \approx G, k_{\mathbb{Z}} Y \approx G$. Next, let $K$ be a compact set of $S$. Since the projection $\pi: S \rightarrow Y$ is continuous, it follows from Claim 2 that $|\pi[K]| \leq \omega$. This implies that the characteristic function of $G$ on $S$ is $k$-continuous. Hence, $k_{\mathbb{Z}} S \approx k_{\mathbb{Z}} G \sqcup k_{\mathbb{Z}} H$. Since $k_{\mathbb{Z}} G=G$ and $H \approx X$, $k_{\mathbb{Z}} S \approx G \sqcup k_{\mathbb{Z}} X$.

Claim 4. $\beta_{\mathbb{Z}} G=S$.

Proof. First, we show that $S$ is $\mathbb{Z}$-compact. Let $p$ be an $\omega_{1}$-complete clopen ultrafilter on $S$. If there is $U \in p$ with $U \cap H=\emptyset$, then $U \subset \pi^{-1}\left[Y_{\mu}\right]$ for some $\mu<\kappa$. Since $\pi^{-1}\left[Y_{\mu}\right] \approx Y_{\mu}, p$ converges by Claim 1. If $U \cap H \neq \emptyset$ for each $U \in p$, put $q=\{U \cap H: U \in p\}$. Observe that (i) for each clopen set $V$ in $H$, there is a clopen set $W$ in $S$ such that $W \cap H=V$ and that (ii) if $U$ is a clopen set in $S$ with $U \cap H \neq \emptyset$, then there is $\nu<\kappa$ such that $((\nu, \kappa] \times\{\lambda \in X:\langle\kappa, \lambda\rangle \in U\}) \cap S \subset U$. (i) implies that $q$ is a clopen ultrafilter on $H$ and (ii) implies that $q$ is $\omega_{1}$-complete. Since $H \approx X$ and $X$ is $\mathbb{Z}$-compact, $q$ converges, and hence, $p$ converges to the same point. Thus, $S$ is $\mathbb{Z}$-compact. Next, we show that each $\mathbb{Z}$-valued continuous function $f$ on $G$ can be continuously extended over $S$. For each $\lambda \in(X \cap \Omega) \cup\{\kappa\}$, put $F_{\lambda}=\left\{\langle\alpha, \varphi(\alpha)\rangle: \alpha \in E_{\lambda}\right\}$. Then the subspace $F_{\lambda}$ of $G$ is homeomorphic to $E_{\lambda}$ with the relative order topology. Since $E_{\lambda}$ is stationary, there is $\beta(\lambda)<\kappa$ such that $f$ takes a constant value $g(\lambda)$ on $\left\{\langle\alpha, \varphi(\alpha)\rangle \in F_{\lambda}: \alpha>\beta(\lambda)\right\}$. We show that $g$ is a continuous function on the subspace $(X \cap \Omega) \cup\{\kappa\}$ of $X$. Applying Forder's lemma to $F_{\kappa}$, we can find $\nu_{0}<\kappa$ such that $f\left[\left(\left(\nu_{0}, \kappa\right] \times\left(\nu_{0}, \kappa\right]\right) \cap G\right]=\{g(\kappa)\}$. Thus, $g(\xi)=g(\kappa)$ for each $\xi \in X \cap \Omega \cap\left(\nu_{0}, \kappa\right]$, which shows that $g$ is continuous at $\kappa$. For $\lambda \in X \cap \Omega$, put $\beta_{0}=\sup _{\xi \leq \lambda} \beta(\xi)$ and $F=\bigcap_{\xi<\lambda} \mathrm{cl} E_{\xi}$, where the closure is 
taken in $\kappa$ with the order topology. Then $F$ is closed and unbounded, and hence, there is $\beta_{1} \in F \cap E_{\lambda} \cap\left(\beta_{0}, \kappa\right]$. Since $f\left(\left\langle\beta_{1}, \lambda\right\rangle\right)=g(\lambda)$, there is a neighborhood $U$ of $\lambda$ in $X$ such that $f\left[\left(\left(\beta_{0}, \beta_{1}\right] \times U\right) \cap G\right]=\{g(\lambda)\}$. By the condition (1) for $X$, we may assume that $U \subset \lambda+1$. Since $\left(\beta_{0}, \beta_{1}\right] \cap E_{\xi} \neq \emptyset$ for each $\xi \in U \cap \Omega$, $g[U \cap \Omega]=\{g(\lambda)\}$. Hence, $g$ is continuous at $\lambda$. By the condition (4) for $X, g$ can be extended to a continuous function $\bar{g}$ on $X$. Extend $f$ over $S$ by letting $f(\langle\kappa, \lambda\rangle)=\bar{g}(\lambda)$ for each $\lambda \in X$. Then, it is easily checked that the extension is continuous. Hence, $\beta_{\mathbb{Z}} G \approx S$.

By Claims 3 and $4, \beta_{\mathbb{Z}} k_{\mathbb{Z}} Y \approx \beta_{\mathbb{Z}} G \approx S$. To prove (c), let $K$ be a compact set of $S$. Then, by Claim $2,|\pi[K]| \leq \omega$, which implies that $K \cap G$ is countable and clopen in $K$. Since each point of $G$ has a countable neighborhood base, $w(K \cap G) \leq \omega$. Since $H \approx X, w(K) \leq k w(X)$, and hence, $k w(S) \leq k w(X)$. Conversely, since $X \approx H \subset S, k w(X) \leq k w(S)$. Thus, we have (c). Finally, by Claim $3, \beta_{\mathbb{Z}} k_{\mathbb{Z}} S \approx$ $\beta_{\mathbb{Z}} G \sqcup \beta_{\mathbb{Z}} k_{\mathbb{Z}} X \approx \beta_{\mathbb{Z}} k_{\mathbb{Z}} Y \sqcup \beta_{\mathbb{Z}} k_{\mathbb{Z}} X$. Since $\beta_{\mathbb{Z}} k_{\mathbb{Z}} Y \approx S$, we have (d), which completes the proof of Lemma 3 .

Lemma 4. Assume that $E(\kappa)$ holds. Then there exists a collection $\left\{Y_{\kappa, n}: n \in \mathbb{Z}\right\}$ of $\mathbb{Z}$-compact spaces such that:

(a') $\beta_{\mathbb{Z}} k_{\mathbb{Z}} Y_{\kappa, n} \approx Y_{\kappa, n+1}$ for each $n \in \mathbb{Z}$

(b') $Y_{\kappa, n}=Y_{\kappa, 1}$ for each $n \geq 1$,

$\left(\mathrm{c}^{\prime}\right) k w\left(Y_{\kappa, n}\right)=\omega$ for each $n<0$, and

$\left(\mathrm{d}^{\prime}\right) k w\left(Y_{\kappa, n}\right)=\kappa$ for each $n \geq 0$.

Before proving Lemma 4, we define some symbols. For a space $X$, let $\bigsqcup_{\omega} X$ denote the discrete sum of $\omega$ many copies of $X$; in other words, $\bigsqcup_{\omega} X=X \times \mathbb{Z}$. We denote the space $Y$ constructed from an adequate space $X$ in Lemma 3 by $\Delta(X)$. Since $\Delta(X)$ is an adequate space again, we can define $\Delta(\Delta(X))$. Inductively, we write $\Delta^{i+1}(X)=\Delta\left(\Delta^{i}(X)\right)$ for each $i \geq 0$, where $\Delta^{0}(X)=X$.

Proof of Lemma 4. Let $X=\kappa+1$ with the order topology. Then $X$ is compact, and hence, $\beta_{\mathbb{Z}} k_{\mathbb{Z}} X=X$ and $k w(X)=\kappa$. Since $X$ is an adequate space, we can define the space $\Delta^{i}(X)$ for each $i \geq 0$. For each $n \in \mathbb{Z}$, define

$$
Y_{\kappa, n}=\bigsqcup_{i \geq m(n)}\left(\bigsqcup_{\omega} \beta_{\mathbb{Z}} k_{\mathbb{Z}} \Delta^{i}(X)\right), \text { where } m(n)=\max \{1-n, 0\}
$$

Then $Y_{\kappa, n}$ is $\mathbb{Z}$-compact and $Y_{\kappa, n}=Y_{\kappa, 1}$ for each $n \geq 1$; thus $\left(\mathrm{b}^{\prime}\right)$ holds. For $n \geq 1$, by (d) in Lemma 3 ,

$$
\begin{aligned}
\beta_{\mathbb{Z}} k_{\mathbb{Z}} Y_{\kappa, n} \approx & \bigsqcup_{\omega} \beta_{\mathbb{Z}} k_{\mathbb{Z}} \beta_{\mathbb{Z}} k_{\mathbb{Z}} \Delta^{0}(X) \\
& \sqcup\left(\bigsqcup_{i \geq 1}\left(\bigsqcup_{\omega}\left(\beta_{\mathbb{Z}} k_{\mathbb{Z}} \Delta^{i}(X) \sqcup \beta_{\mathbb{Z}} k_{\mathbb{Z}} \Delta^{i-1}(X)\right)\right)\right) .
\end{aligned}
$$

Since $\beta_{\mathbb{Z}} k_{\mathbb{Z}} \Delta^{0}(X)=\Delta^{0}(X), \beta_{\mathbb{Z}} k_{\mathbb{Z}} Y_{\kappa, n}$ is homeomorphic to $Y_{\kappa, n+1}$. For $n \leq 0$, 
$m(n)-1=m(n+1)$ and by $(\mathrm{d})$ in Lemma 3 again,

$$
\begin{aligned}
\beta_{\mathbb{Z}} k_{\mathbb{Z}} Y_{\kappa, n} & \approx \bigsqcup_{i \geq m(n)}\left(\bigsqcup_{\omega}\left(\beta_{\mathbb{Z}} k_{\mathbb{Z}} \Delta^{i}(X) \sqcup \beta_{\mathbb{Z}} k_{\mathbb{Z}} \Delta^{i-1}(X)\right)\right) \\
& \approx \bigsqcup_{i \geq m(n+1)}\left(\bigsqcup_{\omega} \beta_{\mathbb{Z}} k_{\mathbb{Z}} \Delta^{i}(X)\right) \approx Y_{\kappa, n+1} .
\end{aligned}
$$

Hence, we have $\left(\mathrm{a}^{\prime}\right)$. By (b) and (c) in Lemma $3, k w\left(\beta_{\mathbb{Z}} k_{\mathbb{Z}} \Delta^{i}(X)\right)=k w\left(\Delta^{i-1}(X)\right)$ $=\omega$ for each $i \geq 2$ and $k w\left(\beta_{\mathbb{Z}} k_{\mathbb{Z}} \Delta^{i}(X)\right)=k w(X)=\kappa$ for $i=0,1$. These imply $\left(\mathrm{c}^{\prime}\right)$ and $\left(\mathrm{d}^{\prime}\right)$.

Proof of Theorem 1. Assume that $E\left(\omega_{i}\right)$ holds for each $1<i<\omega$. Note that $E\left(\omega_{1}\right)$ is always true. By Lemma 4 , for each $i \geq 1$, there exists a collection $\left\{Y_{\omega_{i}, n}: n \in \mathbb{Z}\right\}$ of $\mathbb{Z}$-compact spaces satisfying $\left(\mathrm{a}^{\prime}\right)-\left(\mathrm{d}^{\prime}\right)$. For each $n \in \mathbb{Z}$, define $X_{n}=\bigsqcup_{i \geq 1} Y_{\omega_{i}, n-i}$. Then $X_{n}$ is $\mathbb{Z}$-compact and by $\left(\mathrm{a}^{\prime}\right)$,

$$
\beta_{\mathbb{Z}} k_{\mathbb{Z}} X_{n} \approx X_{n+1} \text { for each } n \in \mathbb{Z}
$$

Moreover, by $\left(c^{\prime}\right)$ and $\left(d^{\prime}\right)$,

$$
k w\left(X_{n}\right)=\omega_{n} \text { for each } n \geq 0,
$$

where $\omega_{0}=\omega$. Put $A_{2 n}=C\left(X_{n}, \mathbb{Z}\right)$ and $A_{2 n+1}=A_{2 n}^{*}$ for each $n \in \mathbb{Z}$. Then, it follows from (6) and Lemma 1 that

$$
\begin{aligned}
A_{2 n+1}^{*} & =C\left(X_{n}, \mathbb{Z}\right)^{* *} \\
& \cong C\left(k_{\mathbb{Z}} X_{n}, \mathbb{Z}\right) \\
& \cong C\left(\beta_{\mathbb{Z}} k_{\mathbb{Z}} X_{n}, \mathbb{Z}\right) \\
& \cong C\left(X_{n+1}, \mathbb{Z}\right)=A_{2 n+2} .
\end{aligned}
$$

If $n \geq 0$, then by (7) and Lemma 2, no subgroup of $A_{2 n+1}$ is isomorphic to $\mathbb{Z}^{\omega_{n+1}}$ but $\bar{A}_{2 n+3}$ has a subgroup isomorphic to $\mathbb{Z}^{\omega_{n+1}}$. Hence, $A_{2 n+1} \nsucceq A_{2 n+3}$ for $n \geq 0$. This means that $A_{n}$ is strongly non-reflexive for each $n \in \mathbb{Z}$.

Proof of Theorem 2. Since $E\left(\omega_{1}\right)$ is always true, there exists a collection $\left\{Y_{\omega_{1}, n}\right.$ : $n \in \mathbb{Z}\}$ of $\mathbb{Z}$-compact spaces satisfying $\left(\mathrm{a}^{\prime}\right)-\left(\mathrm{d}^{\prime}\right)$ by Lemma 4 . For each $n \in \mathbb{Z}$, define $X_{n}=Y_{\omega_{1}, n}$, and put $A_{2 n}=C\left(X_{n}, \mathbb{Z}\right)$ and $A_{2 n+1}=A_{2 n}^{*}$. Then, similarly to the proof of Theorem 1, $A_{n}^{*} \cong A_{n+1}$ for each $n \in \mathbb{Z}$. By $\left(\mathrm{c}^{\prime}\right), k w\left(X_{-1}\right)=\omega$ and by $\left(\mathrm{d}^{\prime}\right)$, $k w\left(X_{0}\right)=\omega_{1}$. Hence, it follows from Lemma 2 that $A_{-1} ¥ A_{1}$. This means that $A_{n}$ is strongly non-reflexive for each $n<0$.

Remark 1. Let $X_{n}$ and $A_{n}(n \in \mathbb{Z})$ be the same as in the proof of Theorem 2 . By the proof of Lemma $3, k_{\mathbb{Z}} X_{n} \neq X_{n}$ for each $n \in \mathbb{Z}$. Hence, it follows from [1, Theorem 4.1] that $A_{n}$ is non-reflexive for all $n \geq 0$ too. However, by $\left(\mathrm{b}^{\prime}\right)$ in Lemma $4, A_{n} \cong A_{n+2}$ for each $n \geq 2$. With some effort, it can also be proved that $A_{n} \cong A_{n+2}$ for $n=0,1$.

Remark 2. Our construction yields another strongly non-reflexive dual group $A=$ $C(Y, \mathbb{Z})$. Let $X=\left\{\omega_{1}\right\} \cup\left\{\alpha<\omega_{1}: \alpha\right.$ is a successor $\}$ with the relative order 
topology. Then $X$ is an adequate space. Define $Y=\beta_{\mathbb{Z}} k_{\mathbb{Z}} \Delta(X)$ and $A=C(Y, \mathbb{Z})$; then $A$ is a dual group by Lemma 1 . Since $k_{\mathbb{Z}} \Delta(X)$ has no partition by $\omega_{1}$ many nonempty clopen sets, it follows from $\left[3\right.$, Theorem 3.3] that $A\left(\cong C\left(k_{\mathbb{Z}} \Delta(X), \mathbb{Z}\right)\right)$ has no subgroup isomorphic to $\mathbb{Z}^{\omega_{1}}$. On the other hand, by Lemma $1, A^{* *} \cong C\left(\beta_{\mathbb{Z}} k_{\mathbb{Z}} Y, \mathbb{Z}\right)$ and by (d) in Lemma $3, \beta_{\mathbb{Z}} k_{\mathbb{Z}} Y \approx Y \sqcup \beta_{\mathbb{Z}} k_{\mathbb{Z}} X$. Since every compact set of $X$ is finite, $k_{\mathbb{Z}} X$ is a discrete space, and hence, so is $\beta_{\mathbb{Z}} k_{\mathbb{Z}} X$. These mean that there is a summand of $A^{* *}$ isomorphic to $\mathbb{Z}^{\omega_{1}}$. Hence, $A$ is strongly non-reflexive. Assuming $E(\kappa), \omega_{1}$ can be replaced by $\kappa$.

\section{REFERENCES}

1. K. Eda and H. Ohta, On abelian groups of integer-valued continuous functions, their $\mathbb{Z}$-duals and $\mathbb{Z}$-reflexivity, Abelian Group Theory (R. Göbel and E. Walker, eds.), Gordon and Breach, London, 1985, pp. 241-257. MR 90f:20081

2. K. Eda, T. Kiyosawa and H. Ohta, N-compactness and its applications, Topics in General Topology (K. Morita and J. Nagata, eds.), North-Holland, Amsterdam, 1989, pp. 459-521. MR 95m:54018

3. K. Eda, S. Kamo and H. Ohta, Abelian groups of continuous functions and their duals, Topology and its Appl. 53 (1993), 131-151. MR 94m:20108

4. P. C. Eklof and A. H. Mekler, Almost Free Modules, Set-theoretic Methods, North-Holland, Amsterdam, 1990. MR 92e:20001

5. P. C. Eklof, A. H. Mekler and S. Shelah, On strongly-non-reflexive groups, Israel J. Math. 59 (1987), 283-298. MR 89c:20080

6. G. Schlitt, Sheaves of abelian groups and the quotients $A^{* *} / A$, J. Algebra 158 (1993), 50-60. MR 94e:20072

Faculty of Education, Shizuoka University, Ohya, Shizuoka, 422 Japan

E-mail address: h-ohta@ed.shizuoka.ac.jp 